\title{
Zukunft nach der Praxis
}

\section{Daniel Oertle}

Dr. med., Hausarzt in Zürich-Albisrieden, Mitglied FMH

\author{
Viele Ärztinnen und Ärzte arbeiten über das Pensionierungsalter hinaus. Soll man? \\ Darf man? "Ärzte sind Heil-Künstler: Künstler werden nie pensioniert», sagen die \\ einen. "Piloten werden auch mit 60 pensioniert», sagen die andern. Nun? Eine \\ Polemik. ${ }^{1}$
}

Ich habe keine Ahnung, was nach der Praxis sein wird. Ich bin jetzt 62 und mitten drin im Gewusel einer Hausarztpraxis. Ich komme jeden Abend wie gekocht nach Hause. Wenn Sie mich nach einem solchen Praxistag fragten, wie ich mir die «Zukunft nach der Praxis» ausmale - dann würde ich Ihnen ohne zu zögern sagen: Ich wünsche mir nur 5 Minuten Ruhe, kein Gstürm, kein Telefon, kein Gejammer, kein IV-Formular, keine Kostengutsprache, nix. Ich wünschte nur etwas Zeit für mich zu haben, mit meiner Frau eine Tasse Kaffee zu trinken, ohne gleich aufzustehen oder wegzulaufen. Ich wollte nur etwas Zeit, um meine alte taube Katze zu streicheln, mit der ich nicht einmal mehr reden kann.

Am 2. März 2021 werde ich 65. Und dann ist Schluss. Was ich dann mit mir mache? Keine Ahnung! Wenn man von etwas keine Ahnung hat, ist es immer gut, eine Task-Force zu gründen, das zeigt Tatkraft. Aus der Task-Force ist dann ein Selbsthilfegrüppli geworden und ich und mich haben viel voneinander gelernt.

Am 2. März 2021 ist Schluss. Ich merke es, wenn ich in ein Flugzeug einsteige: Ich schau mir jedes Mal den Piloten genau an und bin froh, wenn dort vorne kein altes tattriges Greislein sitzt, sondern ein flotter, kesser Mann.

\section{«Am 2. März 2021 werde ich 65. Und dann ist Schluss.»}

Abtreten in Würde. Kürzlich erzählte mir eine Bekannte, wie es einem von uns hoch verehrten Professor ergeht. Als Direktor der Klinik pensioniert, eröffnete er in der Stadt eine Praxis. Dort werkelte er vor sich hin, lange, sehr lange. Bis es nicht mehr ging. Dann brachte man ihn ins Heim. Im Heim fällt der Professor dardurch auf, dass er zu irgendwelchen Tagesoder Nachtzeiten in irgendwelche Zimmer geht und dort Visite macht.

Ich möchte in Würde abtreten.
Am 2. März 2021 ist Schluss. Ich mein es Ernst. Denn: Die Halbwertzeit meines Wissens wird immer kürzer und nähert sich asymptotisch der Null: Eigentlich weiss ich schon jetzt fast nichts mehr.

Ich würde einen Diabetiker noch immer mit Metformin und Sulfonylharnstoffen behandeln, weil ich die pharmagesponserte Fortbildung über die wöchentlich inizierbaren GLP-1-Analoga verpasst habe.

Ich kann den CHA2DS2-VAsc-Score nicht auswendig und denke beim Vorhofflimmern noch immer an Cumarine und den Quick in \%.

Und wenn ich Rheumatoide Arthritis höre, denke ich an Prednison und Methotrexat, weil ich mir all die neuen TNF- $\alpha$-Blocker nicht merken kann.

Ich habe keine Ahnung, was ich meiner Patientin mit BRAC-1-positivem Herceptin-negativem Mamma-Karzinom raten soll. Und wenn ich meinen alten Freund Bernhard in der Onkologie des Zentrumsspitals anrufe, dann weiss er es auch nicht und verweist mich an den Organ-Onkologen XY, der wisse es wahrscheinlich. Kürzlich kam ein 35-jähriger Finanzanalyst einer Privatbank zu mir. Er hatte $10 \mathrm{ml}$ EDTA-Blut an ein Gentech-Institut in San Francisco geschickt und streckt mir einen USB-Stick hin: da drauf sei das Whole-genome-Sequencing. Er wollte wissen, ob er ein erhöhtes Risiko hätte für ein Pankreas-Karzinom, wie sein Onkel, der eben daran starb.

Ein 58-jähriger Monteur, 50 pack-years, sitzt da und berichtet über rezidivierendes thorakales Beklemmungsgefühl. Leider hatte ich das 3. von 5 Modulen der Kardiologie-Rezertifizierung verpasst und durfte darum kein EKG ableiten.

Meine beruflichen Seilschaften bröckeln, meine alten Kumpels quittieren den Dienst, andere sind einfach so gestorben. Zuweisungen werden seltener, meine Meinung ist nicht mehr gefragt.

Ich rede mir zwar ein, ich sei noch nicht dement - aber die Zugriffszeiten auf meine Festplatte werden länger. 
Am 2. März 2021 ist Schluss. Ich mein es Ernst. Und was dann? Wenn ich die Praxistür definitiv hinter mir geschlossen haben werde, was dann? Ich weiss es nicht. Ich werde mir wohl ein Smartphone kaufen müssen oder besser zwei, drei: und wenn ich dann auf Facebook, Twitter, Flickr, Instagram, Snapchat, Musically, WhatsApp und so weiter zugeschaltet bin, habe ich den ganzen Tag zu tun.

Mir wird sterbenslangweilig werden, weil ich gar nicht mehr weiss, was ich will.

Ich weiss erst, was ich nicht will.

«Ich will keine beige Windjacke, keine praktische beige Stretch-Hose, keine trittsicheren Schuhe und kein flottes Stoffkäppli.»

Ich will nicht an all die Publikumsveranstaltungen der Spitäler gehen, zusammen mit den vielen fitten alten Goldenagers und mir auf unbequemen Kirchgemeindehaus-Stühlen Vorträge anhören mit Titeln wie: «Das Gebiss im Alter», «Demenz: die lautlose Gefahr?», «Neue Gelenksprothesen: Ihre Chance!»

Ich will keine beige Windjacke, keine praktische beige Stretch-Hose, keine trittsicheren Schuhe und kein flottes Stoffkäppli, möchte nicht auf eine 10-tägige Donauflussfahrt mit 3-Sterne-Koch und den «Original-Oberkrainer-Buaben».

Überhaupt «Reisen»: Viele Menschen beantworten die Frage, was sie denn nach der Pensionierung zu tun gedenken, mit «Reisen». Ja aber ums Himmels willen, will ich denn reisen? "China in 10 Tagen", «Die Salzwüste von Uyuni», "Jurtentrecking in der Mandschurei», «Unbekanntes Bhutan» - will ich das?

Fotos sammeln wie Paninibildli fürs grosse Welt-Album? Kürzlich trafen wir eine Reisegruppe, die auf ihren Rollkoffern einen Sticker aufgeklebt hatten "ABD: Adventure before Dementia» - meint Reisen das?

Man bekommt ja heute ungefragt von allerhand Leuten travel blogs mit vielen, vielen Bildli. Also zapp ich noch schnell in den Blog von Markus, der in Alaska am Lachsfischen ist, ziehe mir noch ein paar Bildli von Peter rein, der irgendwo in Brasilien rumschippert dann hab ich für diesen Tag genug gereist.

Ich möchte es mit Menachem halten, dem alten Freund meiner Eltern, der jedes Jahr zur selben Zeit von Israel zur selben Reise aufbrach: Er besuchte seine Cousine in London, seinen Freund in Berlin, einen Verwandten in Baden-Baden und dann noch meine Eltern in Zürich.
Jedes Jahr. Mein Vater kaufte jeweils einen Kasten Bier im Discounter, das Bier hiess "Ochsenbier». Menachem schrieb meinen Eltern einige Wochen vor seiner Reise, dass er sich sehr auf den Aufenthalt im Gasthaus «Zum Goldenen Ochsen» freue, und meine Eltern freuten sich sehr. Menachem sagte immer, dass ihm der Besuch seiner Freunde das Wichtigste sei, viel wichtiger als Reisen in fremde Länder mit fremden Menschen in fremden Landschaften.

Menachem kam und setzte sich mit meinen Eltern in die Stube und man redete und lachte und am nächsten Tag wieder, und wenn dann alle Ochsen leer waren ging Menachem und versprach, nächstes Jahr wieder zu kommen. Seine Reisen wurden kürzer, als die Cousine in London starb und später der Verwandte in Baden-Baden - und eines Tages kam Menachem nicht mehr.

Ich möchte so reisen wie Menachem reiste.

Ich will nicht als sogenannter Experte in ein Entwicklungsland gehen, um dort ein Spital aufzubauen an einem Ort, wo ich noch nie war, wo ich die Sprache nicht kenne und wo ich vor allem nicht weiss, ob die Leute dort überhaupt ein Spital haben wollen.

«Meine Frau vermutete schon immer, dass ich nur Arzt geworden sei, um all die Geschichten zu hören ...»

Ich will nicht alt und peinlich werden, meine Ehefrau nach 38 Jahren verlassen, die Haare zu einem HipsterDutt aufstecken, ein Goldketteli und einen Dreitagebart montieren, ein Tattoo und eine viel zu junge Frau am Arm, dazu den wippenden Gang in coolen Sneakers. Oje.

Am 2. März 2021 ist Schluss. Und spätestens am 4. März werde ich ganz traurig werden, Heimweh haben nach meinen wunderbaren Gspänli in der Praxis, nach meinen Kolleginnen und Kollegen, den vielen freundlichen, interessanten und gspunnenen Patienten mit ihren schönen und schrägen Geschichten. Meine Frau vermutete schon immer, dass ich nur Arzt geworden sei, um all die Geschichten zu hören ...

Oje, ich werde dann nur noch ein alter Mann sein, nie mehr der brillante Diagnostiker Dr. House, kein Tröster, kein Held, kein Lehrer.

Wer ich dann bin, weiss ich noch nicht. Ich werde mich neu erfinden müssen. Ich möchte, wenn's geht, fröhlich bleiben. 